\title{
Acute Severe Mitral Regurgitation Secondary to Haemophilus parainfluenzae Infective Endocarditis
}

\author{
Authors: \\ *Brian P. Kelley, ${ }^{1}$ Nathaniel C. Warner, ${ }^{2}$ Sanjay Venkatesh, ${ }^{3}$ Thelsa T. \\ Weickert ${ }^{3}$ \\ 1. Department of Internal Medicine, University of North Carolina Hospitals, Chapel \\ Hill, North Carolina, USA \\ 2. Division of Infectious Disease, University of North Carolina Hospitals, Chapel Hill, \\ North Carolina, USA \\ 3. Division of Cardiology, University of North Carolina Hospitals, Chapel Hill, North \\ Carolina, USA \\ *Correspondence to brian.kelley@unchealth.unc.edu
}

Disclosure: $\quad$ The authors have declared no conflicts of interest.

Received: $\quad 10.06 .20$

Accepted: $\quad 17.08 .20$

Keywords: $\quad$ Haemophilus parainfluenzae, infective endocarditis (IE), native mitral valve, mitral regurgitation (MR).

Citation: $\quad$ EMJ Cardiol. 2020;8[1]:92-96

\section{Abstract}

Haemophilus parainfluenzae is an exceedingly rare cause of infective endocarditis, with only a few case reports describing its potential invasiveness. This case reports on a 25 -year-old female who was admitted with a fever and was subsequently found to have $H$. parainfluenzae endocarditis. She was managed with intravenous antibiotics and mitral valve replacement.

\section{INTRODUCTION}

Haemophilus parainfluenzae has established itself as an important, albeit uncommon, cause of infective endocarditis (IE). In a multinational cohort study conducted in 2013 on patients hospitalised with definite or possible infective endocarditis, only $1.4 \%$ of cases were caused by a Haemophilus species, Aggregatibacter species, Cardiobacterium hominis, Eikenella corrodens, or Kingella species (the HACEK group of bacteria).

Historically, $H$. parainfluenzae endocarditis has been characterised as having a subacute course and usually inflicts disease in those with poor dentition and a history of pre-existing valve disease. ${ }^{2}$ However, after reviewing the literature, a different tale of its pathogenicity has unfolded over the years; it can lead to an invasive disease with a mortality ranging from $10-35 \%$ in patients without any apparent risk factors. ${ }^{3,4}$ Reports of large, destructive vegetations that most often affect the mitral valve have been described. ${ }^{3}$ Additionally, they are frequently complicated by embolic phenomena such as cerebral emboli and splenic infarcts. ${ }^{4}$

The authors present the case of a 25-year-old female with no relevant medical history who was admitted for fever and was found to have acute native valve endocarditis secondary to $H$. parainfluenzae. She underwent successful replacement of the mitral valve. To the best of the authors' knowledge, this is one of the few cases demonstrating $H$. parainfluenzae endocarditis manifesting as acute severe mitral regurgitation (MR). 
Severe acute respiratory syndrome coronavirus-2 (SARS-CoV-2) PCR testing was A previously healthy 25-year-old female was evaluated in the University of North Carolina Hospitals Emergency Department, Chapel Hill, North Carolina, USA. She presented with 1 week of fever, headache, and neck stiffness; she described the headache as "excruciating." Additional symptoms included nausea, anorexia, and an intermittent aching sensation across her precordium that worsened on inspiration. She denied having any rashes, lower extremity swelling, or dyspnoea, and she was without additional gastrointestinal or genitourinary complaints.

The patient had no past medical history. She was up to date on her immunisation schedule and denied intravenous drug use. She also denied recent dental procedures or having come into contact with anyone sick, but did have an 8-year-old son. However, she had not been living with him prior to the onset of her symptoms. She denied any recent travel and had been practicing social distancing as part of the recommended guidelines for the coronavirus disease 2019 (COVID-19) pandemic.

On physical examination, her temperature was $39.4^{\circ} \mathrm{C}$; blood pressure: 94/64 $\mathrm{mm} \mathrm{Hg}$; heart rate: 163 beats per minute; and oxygen saturation: $94 \%$ while breathing ambient air. A physical exam was notable for photophobia, meningismus, and a hyperdynamic precordium with a $3 / 6$ holosystolic murmur, loudest at the apex, radiating to the axilla. There were no embolic phenomena or focal neurologic deficits. Examination of the oropharynx and skin, including the palms, soles, and nails, were normal.

The blood level of haemoglobin was $11.2 \mathrm{~g} / \mathrm{dL}$; white-cell count: $5.8 \times 10^{9} / \mathrm{L}$ (reference range: 4.0-10.0 $\times 10^{9} / \mathrm{L}$ ) with lymphopenia; platelet count: 74,000/ $\mathrm{mm}^{3}$; troponin: $0.062 \mathrm{ng} / \mathrm{mL}$; pro-brain natriuretic peptide: $2490 \mathrm{pg} / \mathrm{mL}$; C-reactive protein: $328.7 \mathrm{mg} / \mathrm{dL}$; venous lactate: $2.6 \mathrm{mmol} / \mathrm{L}$; sodium: $133 \mathrm{mmol} / \mathrm{L}$, potassium: $3.4 \mathrm{mmol} / \mathrm{L}$; chloride: $95 \mathrm{mmol} / \mathrm{L}$; bicarbonate: $22 \mathrm{mmol} / \mathrm{L}$; urea nitrogen: $14 \mathrm{mg} / \mathrm{dL}$; creatinine: $0.64 \mathrm{mg} / \mathrm{dL}$; glucose: $112 \mathrm{mg} / \mathrm{dL}$; total protein: $5.8 \mathrm{~g} / \mathrm{dL}$; albumin: $3.1 \mathrm{~g} / \mathrm{dL}$; total bilirubin: 0.8 $\mathrm{mg} / \mathrm{dL}$; aspartate aminotransferase: $30 \mathrm{U} / \mathrm{L}$; alanine aminotransferase: $36 \mathrm{U} / \mathrm{L}$; and alkaline phosphatase: $183 \mathrm{U} / \mathrm{L}$.

negative, in addition to a respiratory pathogen panel and HIV screening. CT and MRI of the brain were both unremarkable. A lumbar puncture revealed a mild neutrophilic pleocytosis with 39 nucleated cells $/ \mathrm{mm}^{3} ; 75 \%$ neutrophils; 2 red blood cell count $/ \mathrm{mm}^{3}$; protein: $41 \mathrm{mg} / \mathrm{dL}^{2}$ and glucose: $58 \mathrm{mg} / \mathrm{dL}$. The opening pressure was $17 \mathrm{~cm} \mathrm{H}_{2} \mathrm{O}$. Electrocardiography showed sinus tachycardia with normal intervals and no evidence of ST segment changes. A chest X-ray showed clear lung fields.

Given her neurologic complaints and elevated cardiac biomarkers, the patient was admitted to the hospital with a working diagnosis of viral aseptic meningitis with myocarditis. Volume rescucitation was administered, in addition to empiric antimicrobial coverage with intravenous vancomycin, cefepime, and acyclovir. Upon examination, the cardiac murmur could not be reconciled with the working diagnosis and the patient denied a previous history of a cardiac murmur. Therefore, a transthoracic echocardiogram was performed, which revealed moderate $\mathrm{MR}$ with possible mitral valve vegetation.

Later that night, both of the peripheral blood cultures collected on admission became positive; they were reported as gram-negative rods in the electronic medical record, but re-review of the gram stain revealed them to be coccobacillary gram-negative organisms in chains and clusters, suspicious for Haemophilus spp. The following day, the blood cultures speciated $H$. parainfluenzae using the BD Bactec ${ }^{T M}$ (Becton, Dickinson, and Company, Franklin Lakes, New Jersey, USA) continuous blood culture monitoring system. Her antibiotic regimen was subsequently narrowed to ceftriaxone monotherapy. No susceptibility testing was performed.

Two days later, the patient developed respiratory distress and her cardiac murmur dynamically changed. On auscultation, there was a harsh holosystolic murmur heard at the apex with radiation to the left lower sternal border and axilla. A repeat transthoracic echocardiogram showed severe MR and a likely perforation of the anterior $\left(A_{3}\right)$ versus posterior $\left(P_{3}\right)$ leaflet (Figure 1). 

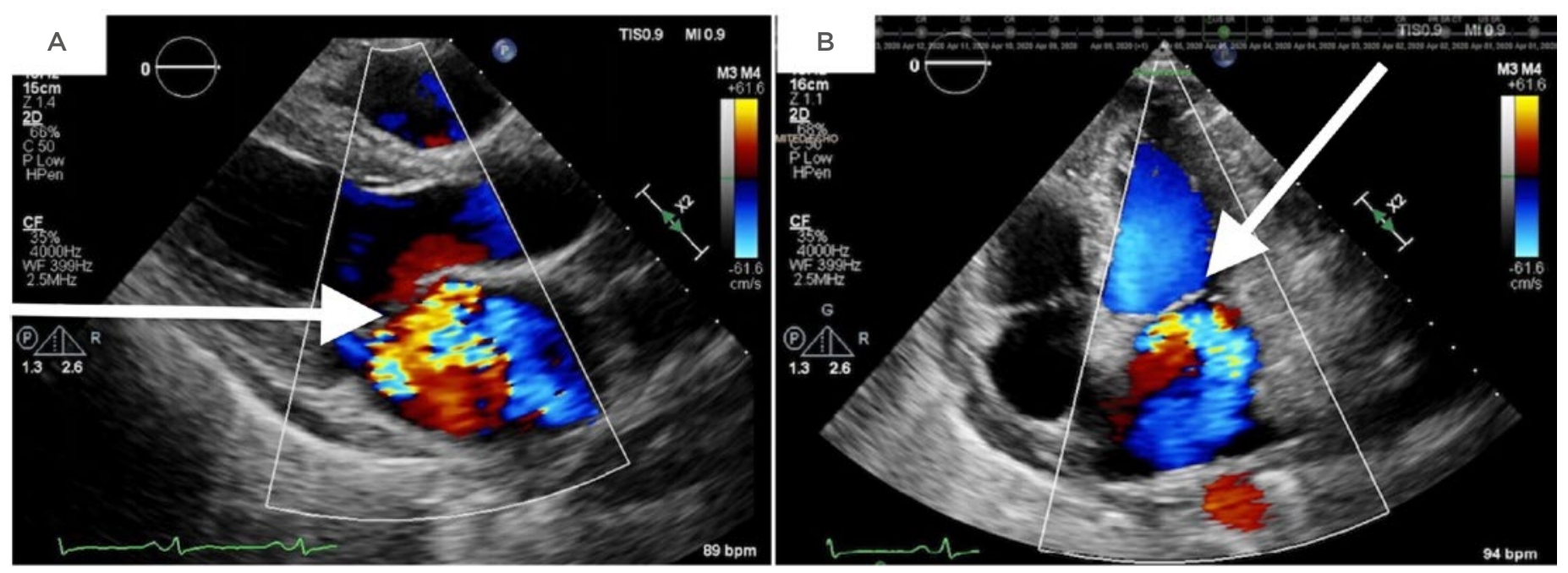

Figure 1: A repeat transthoracic echocardiogram revealing severe mitral regurgitation in the parasternal long-axis view (A) and an apical four-chamber view (B).
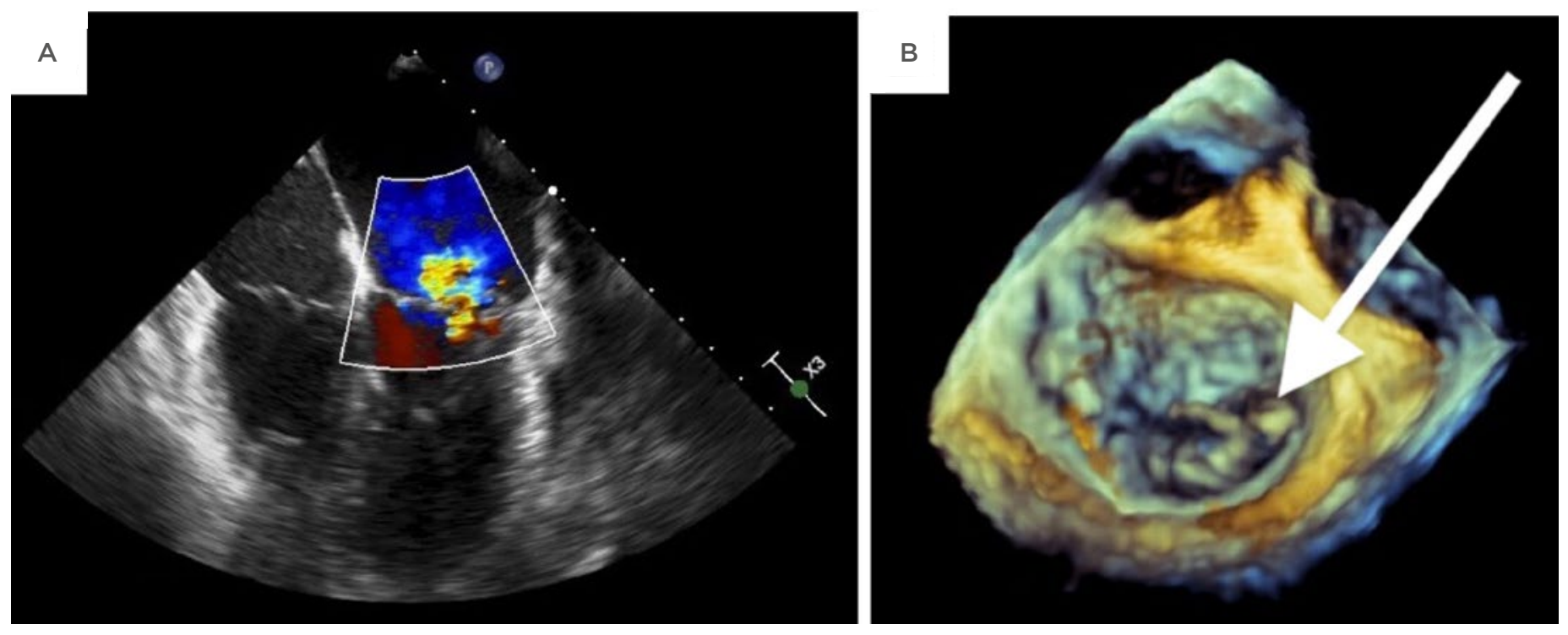

Figure 2: An intraoperative transesophageal echocardiogram demonstrating mitral regurgitation on midesophageal four-chamber view (A) as well as a 3-dimensional transthoracic echocardiogram showing a flail posterior leaflet (B).

Cardiothoracic surgery was considered. Although transesophageal echocardiography was desired for further surgical planning, it was ultimately not pursued because of the risk of periprocedural haemodynamic collapse. She was moved to the cardiac intensive care unit for closer monitoring until she underwent valve replacement. Although her presenting symptoms had mostly resolved, she continued to have sinus tachycardia with intermittent episodes of headache and fever leading up to the date of surgery.
Intraoperative findings included a perforated $A_{2}$ leaflet, a flail $P_{1}$ leaflet, and vegetations involving $\mathrm{P}_{1}, \mathrm{P}_{3}$, and the annulus (Figure 2). The involved leaflets, subvalvular apparatus, annulus, and a portion of the ventricle were resected and replaced with a mechanical mitral valve. The majority of its chordae tendineae were retained.

Although bacterial, fungal, and acid-fast bacilli cultures of the surgical specimens were negative, 16S rRNA sequencing of the valvular tissue revealed $H$. parainfluenzae. Repeat blood 
cultures throughout the rest of her admission remained negative. The patient was started on warfarin with a goal international normalised ratio of 2.5-3.5. She completed 4 weeks of intravenous ceftriaxone with an adjunctive 10 days of gentamicin ( 5 days) followed by levofloxacin (5 days). Her post-discharge course was complicated by postoperative pericarditis treated with colchicine and transient neutropenia in the last week of ceftriaxone which recovered after completion of therapy.

\section{DISCUSSION}

The patient presented with multiple systemic complaints, namely fever, which is seen in $80 \%$ of cases of IE. ${ }^{5}$ Her symptoms of headache, meningismus, and fever led the authors to initially consider meningitis. The prominent murmur and elevated cardiac biomarkers, in combination with echocardiography and blood cultures, were the clues that she did not fit the working diagnosis of aseptic meningitis and ultimately led to the correct diagnosis of IE secondary to $H$. parainfluenzae. However, it should be noted that meningitis can occur as a complication in $3.5 \%$ of IE cases. ${ }^{6}$ The patient was successfully managed with surgical replacement of the mitral valve and intravenous antibiotics. Although not recommended under current American Heart Association (AHA) guidelines, the infectious disease consultants recommended adjunctive therapy with gentamicin and levofloxacin while awaiting clinical improvement.

In addition to the nonspecific symptomatology on presentation, this case was made additionally challenging given the absence of risk factors in the patient. Interestingly, there have been several similar case reports over recent years (Table 1). Faure et al. ${ }^{7}$ describe a young healthy female who presented with fevers, headache, and meningismus. ${ }^{7}$ She was also eventually diagnosed with $H$. parainfluenzae endocarditis and underwent mitral valve repair. This report was helpful because the patient's initial course was very unclear and was made urgent by the severity of her illness. As in this case, careful physical examination and serial echocardiograms were required to demonstrate the presence of IE secondary to $H$. parainfluenzae and the progression of the mitral valvulopathy.

Table 1: Comparison of three patients with Haemophilus parainfluenzae endocarditis.

\begin{tabular}{|c|c|c|c|c|c|}
\hline Age/sex & Location & $\begin{array}{c}\text { Relevant laboratory } \\
\text { tests }\end{array}$ & Echocardiography & Head imaging & Treatment \\
\hline $\begin{array}{l}\text { 25-year-old } \\
\text { female }^{9}\end{array}$ & $\begin{array}{l}\text { Chapel Hill, } \\
\text { North Carolina, } \\
\text { USA }\end{array}$ & $\begin{array}{l}\text { Thrombocytopenia } \\
\text { Normal white blood } \\
\text { cells } \\
\text { Elevated CRP }\end{array}$ & $\begin{array}{l}\text { Mitral valve } \\
\text { vegetation with } \\
\text { perforation }\end{array}$ & Unrevealing & $\begin{array}{l}\text { Mitral valve } \\
\text { replacement } \\
\text { 4-week course of } \\
\text { IV ceftriaxone }\end{array}$ \\
\hline $\begin{array}{l}\text { 33-year-old } \\
\text { female }^{7}\end{array}$ & Lille, France & $\begin{array}{l}\text { Thrombocytopenia } \\
\text { Leukopenia } \\
\text { Elevated CRP }\end{array}$ & $\begin{array}{l}\text { Mitral valve } \\
\text { vegetation }\end{array}$ & Unrevealing & $\begin{array}{l}\text { Mitral valve repair } \\
6 \text {-week course } \\
\text { of IV ceftriaxone, } \\
\text { rifampicin, and } \\
\text { ciprofloxacin }\end{array}$ \\
\hline $\begin{array}{l}\text { 27-year-old } \\
\text { male }^{9}\end{array}$ & $\begin{array}{l}\text { Brooklyn, New } \\
\text { York, USA }\end{array}$ & $\begin{array}{l}\text { Thrombocytopenia } \\
\text { Leukocytosis } \\
\text { Elevated CRP }\end{array}$ & $\begin{array}{l}\text { Mitral valve } \\
\text { vegetation with } \\
\text { perforation }\end{array}$ & $\begin{array}{l}\text { Cerebral emboli } \\
\text { Maxillary sinusitis }\end{array}$ & $\begin{array}{l}\text { Mitral valve repair } \\
\text { 6-week course of } \\
\text { IV ceftriaxone }\end{array}$ \\
\hline
\end{tabular}

All three patients presented with acute onset of fever, chills, and myalgias.

'The authors' case.

CRP: C-reactive protein; IV: intravenous. 
H. parainfluenzae, a gram-negative coccobacillus, is part of the normal flora of the oropharynx. Though rare, it is a member of the HACEK group of bacteria, which are known to cause endocarditis in approximately $3 \%$ of cases. $^{8}$ Because of their oropharyngeal affiliation, HACEK endocarditis has been associated with risk factors such as dental work and nasopharyngeal infections. ${ }^{9}$ The literature describing $H$. parainfluenzae endocarditis is sparse, but there are two reviews of $H$. parainfluenzae endocarditis that describe it as having a subacute onset ( $<2$ months) and a predominance in young people, with a median age of onset of 27 years. Over half of the affected patients had no underlying valvular disease, the mitral valve was most commonly affected, and no portal of entry was identified in $80 \%$ of cases. ${ }^{3,4,7-10}$

H. parainfluenzae endocarditis has been associated with severe complications, including cerebral emboli and mitral valve perforation. ${ }^{6}$ Given this pathogen's propensity to embolise, close attention should be paid to any new neurologic symptoms with a low threshold for neuroimaging to evaluate for a microbial aneurysm or embolism. Although no embolic phenomena were evident on imaging, this case supports its potential for leading to severe destruction of the valvular apparatus. Clinicians should pay particular attention to any new symptoms or dynamic changes to a patient's cardiac exams as this may indicate clinical deterioration and should prompt urgent echocardiography, as well as the early involvement of cardiothoracic surgery. Intraoperatively, the patient was found to have significant deterioration of the mitral valve which necessitated resection of most of the leaflets. There was also erosion into the annulus and ventricle requiring debridement.

\section{CONCLUSION}

This case describes a young female with no previous valvular heart disease who presented with aseptic meningitis and a dynamic murmur on cardiac exam who was ultimately found to have native mitral valve endocarditis as a result of $H$. parainfluenzae. Her course was complicated by acute severe MR requiring mechanical valve replacement. The extensive destruction of the patient's valve highlights the pathogen's virulence. $H$. parainfluenzae endocarditis remains an elusive disease entitity, and though rare, in the setting of culture-negative meningitis with new valvular dysfunction, the diagnosis should be considered by obtaining blood cultures and urgent echocardiography.

\section{References}

1. Chambers ST et al. HACEK infective endocarditis: characteristics and outcomes from a large, multi-national cohort. PLoS One. 2013;8(5):e63181.

2. Jensek JG et al. Haemophilus parainfluenzae endocarditis. Am J Med. 1979;66(1):51-7.

3. Christou L et al. Acute Haemophilus parainfluenzae endocarditis: a case report. J Med Case Rep. 2009;3:7494.

4. Maleka M et al. Acute Haemophilus parainfluenzae infective endocarditis. Ann Clin Case Rep. 2019;4:1679.
5. Cahill TJ, Prendergast BD. Infective endocarditis. Lancet. 2016;387(10021):882.

6. Alkan $\mathrm{G}$ et al. Tricuspid valve infective endocarditis associated with aseptic meningitis: a rare presentation in a child. Arch Ar-gent Pediatr. 2020;118(1):e22-5.

7. Faure E et al. Haemophilus parainfluenzae endocarditis in young adults. J Med Mal. 2016;47(1):58-60.

8. Baddour LM et al. Infective endocarditis in adults: diagnosis, antimicro-bial therapy, and management of complications. Circulation. 2015;132(15):1435-86

9. Duzenli AE et al. Haemophilus parainfluenzae endocarditis associated with maxillary sinusitis and complicated by cerebral emboli in a young man. J Investig Med High Impact Case Rep. 2017;5(2):1-3.

10. Bridwell RE et al. Multisystem organ failure secondary to Haemophilus parainfluenzae infective endocarditis on an ICD lead: a case report. Am J Emerg Med. 2019;37(8):1602. 